DEMONSTRATIO MATHEMATICA

Vol. XXVIII No $2 \quad 1995$

Sławomir Krzyśka

\title{
ON THE EXISTENCE OF CONTINUOUS SOLUTIONS OF URYSOHN AND VOLTERRA INTEGRAL EQUATIONS IN BANACH SPACES
}

\section{Introduction}

In this paper using measure of weak noncompactness developed by de Blasi [5] we prove some existence theorems for the Urysohn integral equation

$$
x(t)=p(t)+\lambda \int_{I} f(t, s, x(s)) d s,
$$

and for the Volterra integral equation

$$
x(t)=p(t)+\int_{0}^{t} f(t, s, x(s)) d s,
$$

where $I=[0, d]$ is a compact interval in $R, f, p$ and $x$ are functions with values in a Banach space $E$ and the integrals are Pettis integrals (for the definitions see [8], [15], [1]).

There have appeared a lot of papers using the measure of weak noncompactness in proving existence theorems for ordinary differential equations.

For the weak solutions if $f$ is only assamed weakly-weakly continuous, it has been shown that weak weak continuity of the right side is insufficient for the existence of weak solutions [6].

Definition. Let $A$ be a bounded nonvoid subset of $E$. The measure of weak noncompactness $\beta(A)$ is defined by

$$
\beta(A)=\inf \left\{t>0: \text { there exists } C e K^{w} \text { such that } A \subset C+t B_{0}\right\},
$$

where $K^{w}$ is the set of weakly compact subset of $E$ and $B_{0}$ is the unit ball. 
The properties of measure of weak noncompactness $\beta$ are analogous to the properties of Kuratowski measure of noncompactness (see [5], [12]).

In this paper we will apply the following theorems.

Theorem 1 [11]. Let $E$ be a metrizable locally convex topological vektor space and let $D$ be a closed convex subset of $E$, and let $F$ be a weakly sequentially continuous map of $D$ into itself. If for some $x \in D$ the implication

(*) $\quad \bar{V}=\overline{\operatorname{conv}}(\{x\} \cup F(V)) \Rightarrow V$ is relatively weakly compact, holds for every subset $V$ of $D$, then $F$ has a fixed point.

THEOREM 2 [12]. Let $H$ be a bounded, equicontinuous subset of $C(I, E)$. Then $\beta(H)=\sup _{t \in I} \beta(H(t))=\beta(H(I))$.

\section{The Uryshon integral equation}

Consider the integral equation (1) with the following assumptions:

$\left(1^{0}\right) \quad p$ is a continuous function from $I$ into $E$;

$\left(2^{0}\right) \quad(t, s, x) \rightarrow f(t, s, x)$ is a function from $I^{2} \times E$ into $E$

which satisfies the following conditions:

(i) for each $(t, s) \in I^{2}, f(t, s, \cdot)$ is weakly-weakly sequentially continuous,

(ii) for each strongly continuous function $x: I \rightarrow E, f(\cdot, \cdot, x(\cdot))$ is Pettisintegrable on $I$,

(iii) for any $h>0$ there exists a mesaurable function $m_{h}: I^{2} \times R_{+}$, such that $\|f(t, s, x)\| \leq m_{h}(t, s)(t, s \in I,\|x\| \leq h)$ and $\int_{I} m_{h}(t, s) d s \leq$ $a(h)<\infty$,

(iv) for any $h>0$ there is a function $d_{h}: I^{3} \rightarrow R_{+}$such that $\| f(t, s, x)-$ $f(\tau, s, x) \| \leq d_{h}(\tau, t, s)(\tau, t, s \in I,\|x\| \leq h)$ and $\lim _{t \in \tau} \int_{I} d_{h}(\tau, t, s) d s$ $=0$.

Theorem 3. Assume, in addition to $1^{0}$ and $2^{0}$, that there exists an integrable function $k: I^{2} \times R_{+}$such that for every $t \in I, \varepsilon>0$ and for every bounded subset $X$ of $E$ there exists a closed subset $I_{\varepsilon}$ of $I$ such that $\operatorname{mes}\left(I \backslash I_{\varepsilon}\right)<\varepsilon$ and

$$
\beta(t, T \times X) \leq \sup _{s \in T} k(t, s) \beta(x)
$$

for any compact subset $T$ of $I_{\varepsilon}$.

Then there exists $\varrho>0$ such that for each $\lambda, 0 \leq \lambda \leq \varrho$ there exists at least one continuous solution of (1). 
P r o of. Denote by $C$ the Banach space of continuous functions $u: I \rightarrow E$ with the usual supremum norm \|\|$_{C}$. Let $r\left(K^{\prime}\right)$ be the spectral radius of the integral operator $K$ defined by

$$
K u(t)=\int_{I} k(t, s) u(s) d s \quad(u \in C, t \in I) .
$$

Put

$$
\varrho=\min \left(\sup \frac{h-\|p\|_{C}}{a(h)}, \frac{1}{r(K)}, \frac{1}{d}\right) .
$$

For fixed $\lambda \in R, 0 \leq \lambda<\varrho$, choose $b>0$ in such a way that

$$
\|p\|_{C}+\lambda a(b) \leq b .
$$

Put $B=\left\{x \in C:\|x\|_{C} \leq b\right\}$. Consider the operator $G$ defined by

$$
G(x)(t)=p(t)+\lambda \int_{I} f(t, s, x(s)) d s \quad(x \in B, t \in I) .
$$

Because for $x^{*} \in E^{*}$ with $\left\|x^{*}\right\| \leq 1$ and $x \in B$ by (4) we have

$$
\begin{aligned}
\left|x^{*}(G(x)(t))\right| & \leq\left|x^{*}(p(t))\right|+|\lambda| \int_{I} \mid x^{*}(f(t, s, x(s)) \mid d s \leq \\
& \leq\|p\|_{C}+|\lambda| \int_{I}\|f(t, s, x(s))\| d s \leq \\
& \leq\|p\|_{C}+|\lambda| \int_{I} m_{b}(t, s) d s \leq\|p\|_{C}+|\lambda| a(b) \leq b .
\end{aligned}
$$

Consequently

$$
\sup \left\{\left|x^{*}(G(x)(t))\right|: x^{*} \in E^{*},\left\|x^{*}\right\| \leq 1\right\}=\|G(x)(t)\| \leq b .
$$

Also

$$
\begin{aligned}
& \left|x^{*}(G(x)(t)-G(x)(\tau))\right| \\
& \quad \leq\left|x^{*}(p(t)-p(\tau))\right|+\lambda \int_{I}\left|x^{*}[f(t, s, x(s))-f(\tau, s, x(s))]\right| d s \leq \\
& \quad \leq\|p(t)-p(\tau)\|+\lambda \int_{I}\|f(t, s, x(s))-f(\tau, s, x(s))\| d s \leq \\
& \quad \leq\|p(t)-p(\tau)\|+\lambda \int_{I} d_{h}(\tau, t, s) d s .
\end{aligned}
$$


This implies that

$$
\|G(x)(t)-G(x)(\tau)\| \leq\|p(t)-p(\tau)\|+\lambda \int_{I} d_{h}(\tau, t, s) d s .
$$

The assumptions $1^{0}, 2^{0}$ and (5), (6) imply that $G$ is a continuous mapping from $B$ into itself and $\mathrm{G}(\mathrm{B})$ is strongly equicontinuous subset of $B$.

Since $F(t, s, \cdot)$ is weakly-weakly sequentially continuous, by using the Lebesgue dominated convergence theorem, for each $x^{*} \in E^{*}$.

$$
x^{*}\left(G\left(x_{n}\right)(t)\right) \rightarrow x^{*}(G(x)(t)) \quad \text { whenever } x_{n} \rightarrow x \text { in }(C(I, E), \omega) .
$$

So by Lemma 1.9 [12] $G$ is weakly-weakly sequentially continuous.

Let $\nabla=\overline{\operatorname{conv}}(G(V) \cup\{0\})$. Obviously

$$
V(t) \subset \overline{\operatorname{conv}}(G(V)(t) \cup\{0\}) \quad \text { for } t \in D .
$$

Since $V$ is equicontinuous, the function $t \rightarrow V(t)=\beta(V(t))$ is continuous on $I$.

Fix $t \in D$ and $\varepsilon>0$. By (3) and the Lusin theorem there exists a compact subset $I_{\varepsilon}$ of $I$ such that $\operatorname{mes}\left(I \backslash I_{\varepsilon}\right)<\varepsilon$ and $\beta(f(t, T \times X) \leq$ $\sup _{s \in T} k(t, s) \beta(X)$ for any compact subset $T$ of $D_{\varepsilon}$, while the function $s-k(t, s)$ is continuous and

$$
\lambda \int_{I \backslash I_{c}} m_{b}(t, s) d s<\frac{\varepsilon}{2} .
$$

We devide the interval $I=[0, d]$ into $n$ parts $0=d_{0}<d_{1}<\ldots<d_{n}=d$ in such a way that

$$
|k(t, s) V(r)-k(t, u) V(z)|<\varepsilon \quad \text { for } s, r, u, z \in T_{i}=D_{i} \cap D_{\varepsilon},
$$

where $D_{i}=\left[d_{i-1}, d_{i}\right](i=1, \ldots, n)$.

Set $V_{i}=\left\{u(s): u \in V, s \in D_{i}\right\}$, then

(7) $\beta\left(p(t)+\lambda \int_{I} f(t, s, V(s)) d s\right)$

$$
\begin{aligned}
& \leq \beta\left(p(t)+\lambda \int_{I_{c}} f(t, s, V(s)) d s+\lambda \int_{I \backslash I_{c}} f(t, s, V(s)) d s\right) \leq \\
& \leq \beta\left(\lambda \int_{I_{c}} f(t, s, V(s)) d s\right)+\varepsilon .
\end{aligned}
$$


Let us observe that

$$
\begin{aligned}
& \lambda \int_{I_{c}} f(t, s, V(s)) d s \subset \sum_{i=1}^{n} \lambda \int_{T_{i}} f(t, s, V(s)) d s \subset \\
& \subset \lambda \sum_{i=1}^{n} \operatorname{mes} T_{i} \overline{\operatorname{conv}} f\left(t, T_{i} \times V_{i}\right) .
\end{aligned}
$$

By the properties of measure of weak noncompactness we have

$$
\begin{aligned}
\beta\left(\lambda \int_{I_{i}} f(t, s, V(s))\right) d s & \leq \beta\left(\lambda \sum_{i=1}^{n} \operatorname{mes} T_{i} \overline{\operatorname{conv}} f\left(t, T_{i} \times V_{i}\right)\right) \leq \\
& \leq \lambda \sum_{i=1}^{n} \operatorname{mes} T_{i} \beta\left(f\left(t, T_{i} \times V_{i}\right)\right) \leq \\
& \leq \lambda \sum_{i=1}^{n} \operatorname{mes} T_{i} \sup _{s \in T_{i}} k(t, s) \beta\left(V_{i}\right)= \\
& =\lambda \sum_{i=1}^{n} \operatorname{mes} T_{i} k\left(t, q_{i}\right) V\left(s_{i}\right),
\end{aligned}
$$

where $q_{i} \in T_{i}, s_{i} \in D_{i}$. Moreover, as

$$
\left|k(t, s) V(s)-k\left(t, q_{i}\right) V\left(s_{i}\right)\right|<\varepsilon
$$

for $s \in T_{i}$, we have

$$
\operatorname{mes} T_{i} k\left(t, q_{i}\right) V\left(s_{i}\right) \leq \int_{T_{i}} k(t, s) V(s) d s+\varepsilon \operatorname{mes} T_{i} .
$$

Thus

$$
\beta\left(\lambda \int_{I_{\mathbf{t}}} f(t, s, V(s)) d s\right) \leq \lambda \int_{I_{\mathrm{c}}} k(t, s) d s+\lambda \varepsilon \operatorname{mes} T_{\varepsilon} .
$$

As $\varepsilon$ is arbitrarily small, from this and (7) we deduce that

$$
\beta\left(p(t)+\lambda \int_{I} f(t, s, V(s) d s) \leq \lambda \int_{I} k(t, s) V(s) d s\right.
$$

and therefore

$$
\beta(V(t)) \leq \lambda \int_{I} k(t, s) V(s) d s
$$

Because this inequality holds for every $t \in I$ and $\lambda r\left(K^{*}\right)<1$, by applying the theorem on integral inequalities, we conclude that $\beta(V(t))=0$ for $t \in I$. 
By Theorem 2, $V$ is relatively weakly compact in $C(I, E)$. Applying now Theorem 1 we conclude that $G$ has a fixed point, which is a solution of the equation (1).

\section{The Volterra integral equation}

Consider now the integral equation (2) assuming that $p$ and $f$ satisfy $1^{0}$ and $2^{0}$. Choose $b>0$ in such a way that $b>2 \sup _{t \in I}\|p(t)\|$. From $2^{0}$ (iii) it follows that there is number $a, 0<a \leq d$ such that

$$
\int_{0}^{t} m_{b}(t, s) d s \leq \frac{b}{2} \quad \text { for } 0 \leq t \leq a .
$$

Let $J=[0, a]$. Put $B=\left\{u \in C(J, E):\|u\|_{C} \leq b\right\}$ and

$$
F(x)(t)=p(t)+\int_{0}^{t} f(t, s, x(s)) d s \quad \text { for } x \in B, t \in J .
$$

Similarly to the Urysohn integral equation, we can show that $F$ is a weakly-weakly sequentially continuous mapping and the set $F(B)$ is equiuniformly continuous.

Further, let $P=\left\{(t, s, z) \in R^{3}: 0 \leq s \leq t \leq l,|z|<C\right\}$, where $l>a$, $c>2 b$. Assume that a nonnegative real-valued function $(t, s, z) \rightarrow h(t, s, z)$ defined on $P$ is a Kamke function, i.e. $h$ satisfies the Caratheodory conditions and $2^{0}$ (iii)-(iv), and

(v) for each fixed $(t, s)$ the function $z \rightarrow h(t, s, z)$ is nondecreasing,

(vi) for each $q, 0 \leq q \leq l$, the zero function is the unique continuous solution of the equation

$$
z(t)=\int_{0}^{t} h(t, s, z(s)) d s \quad \text { defined on }[0, q] .
$$

Theorem 3. Assme that for any $\varepsilon>0$, bounded $X \subset E$ and $t \in J$ there exists a closed subset $I_{\varepsilon}$ of $[0, t]$ such that mes $\left([0, t] \backslash I_{\varepsilon}\right)<\varepsilon$ and

$$
\beta(f(t, T x X)) \leq \sup _{s \in T} h(t, s, \beta(X))
$$

for each closed subset $T$ of $I_{\varepsilon}$. Then the equation (2) has at least one continuous solution on $J$.

P r o of. Let $V \subset B$ be such that $\nabla=\overline{\operatorname{conv}}(F(V) \cup\{0\})$. Let as fix $t \in J$, $\varepsilon>0$.

By the Scorza Dragoni theorem there exists a closed subset $D_{\varepsilon}$ of $J$ such that $\operatorname{mes}\left(J \backslash D_{\varepsilon}\right)<\varepsilon$ and the function $h$ is uniformly continuous on 
$D_{\varepsilon} \times\left[0, b_{1}\right]$, where $b_{1}=b \beta\left(K^{-}(B(J))\right)$. Analogously as in [7] we prove that

$$
\beta(V(t)) \leq \int_{0}^{t} h(t, s, V(s)) d s \quad \text { for } t \in J .
$$

From the property of Kamke functions and the theorem on integral inequalities, we conclude that

$$
\beta(V(t))=0 \quad \text { for } t \in J .
$$

Now as in the proof of Theorem 2 we conclude that $F$ has a fixed point.

$\mathrm{R}$ e m ark. An analogous theorem can be proved for axiomatic measures of weak noncompactness (see [2], [7]).

\section{References}

[1] O. Arino, S. Gautier, J. P. Penot, A fixed point theorem for sequentially continuous mappings with application to ordinary differential equations, Funkcial. Ekvac. 27 (1984), 273-279.

[2] J. Ban aś, J. Rivero, On measure of weak noncompactness, Ann. Mat. Pura Appl. 125 (1987), 213-224.

[3] M. Cichoń, On bounded weak solutions of a nonlinear differential equation in Banach spaces, Funct. Approximatio 21 (1992), 27-35.

[4] E. Cramer, V. Lakshmikanth am, A. R. Mitchell, On the existence of weak so. lutions of differential equations in nonreflexive Banach spaces, Nonlin. Anal. Theory Meth. Appl. 2 (1978), 169-177.

[5] F. S. De Blasi, On a property of the unit sphere in a Banach Space, Bull. Math. Soc. Sci. Math. R.S. Roumanie 21 (1977) 259-262.

[6] G. D. Faulkner, On the nonexistence of weak solutions to abstract differential equations in nonreflexive spaces, Nonlinear Anal. 2 (1978), 505-508.

[7] J. Januszewski, On the existence of continuous solutions of nonlinear integral equations in Banach spaces, Comment. Math. Prace Mat. 30 (1990), 85-92.

[8] W. J. Knight, Solutions of differential equations in B-spaces, Duke Math. J. 41 (1974), 437-442.

[9] I. Ku bi acz y k, Kneser type theorems for ordinary differential equations in Banach spaces, J. Differential Equations 45 (1982), 139-146.

[10] I. Kubiaczyk, S. Sz ufla, hineser's theorem for weak solutions of ordinary differential equations in Banach spaces, Publ. Inst. Mat. Beograd 32 (1982), 99-103.

[11] I. Kubiaczyk, On a fixed point theorem for weakly sequentially continuous mapping, (in print).

[12] A. R. Mitchell, Ch. Smith, An existence theorem for weak solutions of differential equations in Banach spaces, in Nonlinear Equations in Banach Spaces, ed. V. Lakshmikantham 1978.

[13] A. Szep, Existence theorem for weak solutions of ordinary differential equations in reflexive Banach spaces, Studia Sci. Math. Hungar 6 (1971), 197-203. 
[14] S. Szufla, Kneser's theorem for weak solutions of ordinary differenlial equations in reflexive Banach spaces, Bull. Polon. Sci. Math. 26 (1978), 407-413.

[15] M. Talagrand, Pettis inlegral and measure theory, Memoires Amer. Math. Soc. 307 vol.51, Amer. Math. Soc., Providence, Rhode Island (1984).

INSTITUTE OF MATHEMATICS

ADAM MICKIEWICZ UNIVERSITY

ul. Matejki 48/49

60-769 POZNAÑ, POLAND

Received May 10, 1993. 